\title{
ACCELERATION OF UNHAIRING USING A DIFFERENCE OF PRESSURES
}

Valeika V. ${ }^{1}$

Kaunas University of Technology, Kaunas, Lithuania

The method of accelerated unhairing without degrading the hair was studied. The use of vacuum eliminated the influence of thickness on the penetration of unhairing solution. After speeding up the unhairing by vacuum and 12 hours of keeping of samples the strength of bond between hair and derma did not exceed 0,98 N. It means that hair can be removed by special mechanical means.

Keywords: hide, unhairing, vacuum, penetration, derma.

\section{Introduction}

The duration of most leather manufacture processes is determined by the duration of penetration of chemicals through the skin layers, as the interaction of chemicals with the dermis is much faster than those substances are absorbed into the skin. For this reason, a lot of effort is being put into developing methods or equipment for hide/leather treatment that would speed up the penetration of solutions into the skin.

The various means have been taken to achieve one's object. The use of ultrasound [1-4], hydro acoustics impulses [5], vacuum [6], pressure [7] or mechanical action [8] could be mentioned for example. The survey of mentioned investigations has shown that the efficiency of stimulation method depends on the process, which is carried out.

It was determined that the duration of absorption of sodium sulphide and sodium hydroxide solution into the hide is about $2 \mathrm{~h}$, meanwhile the bond of hair with the dermis is weakened much faster. To accelerate the penetration of materials, it is proposed to use an electric field in which $\mathrm{S}^{2-}$ and $\mathrm{OH}^{-}$ions are drawn on the anode, thus reducing the absorption time from 140 to $10-20 \mathrm{~min}$. [9].

The aim of this study was to investigate the method of accelerated unhairing without degrading the hair, when the unhairing solution is forced to diffuse into the dermis by creating a pressure difference on both sides of the treated samples, as well as to examine the properties of the obtained pelt.

Virgilijus Valeika - Dr., prof. of Department of Polymer Chemistry and Technology, Tel.: +37037300189, e-mail: virgilijus.valeika@ktu.1t 


\section{Experimental}

Salted cattle hides was used as raw material. The samples characterised by various thickness (thickness nearest to $-3,5 ; 5 ; 7$ and $9 \mathrm{~mm}$ ) for the investigation were taken from lower part of hide and cut into pieces $8 \times 12$ $\mathrm{cm}$, and experimental series were prepared from them. All samples were washed and soaked as follows: washing: $\mathrm{H}_{2} \mathrm{O} 200 \%$ (percents from hide mass), temperature $25^{\circ} \mathrm{C}$, duration 1 hour, run continuously; soaking: $\mathrm{H}_{2} \mathrm{O}$ $200 \%$, temperature $25^{\circ} \mathrm{C}, \mathrm{Na}_{2} \mathrm{CO}_{3} 1,5 \%, \mathrm{Na}_{2} \mathrm{SiF}_{6} 0,1 \%$, duration $10 \mathrm{~h}$, run continuously first 1.5 hour, 2 hours in peace, later 6.5 hours run continuously.

A solution with the following composition was used to study the unhairing process: $\mathrm{NaCl} 80 \mathrm{~g} / \mathrm{l}, \mathrm{NaOH}(100 \%)$ - $100 \mathrm{~g} / \mathrm{l}, \mathrm{Na}_{2} \mathrm{~S}$ (100\%) - $80 \mathrm{~g} / \mathrm{l}$. The samples were treated by the solution in two ways.

Method I (treatment parameters in this method were selected after preliminary tests): $10 \mathrm{ml}$ of the solution is poured on the subcutaneous side of the samples and the solution is sucked through the sample $(0,08 \mathrm{MPa})$ for 10 minutes using a special vacuum pressure device (Fig. 1). The samples are placed in a polvethvlene bag and kent for 0 to 12 hours (test samples).

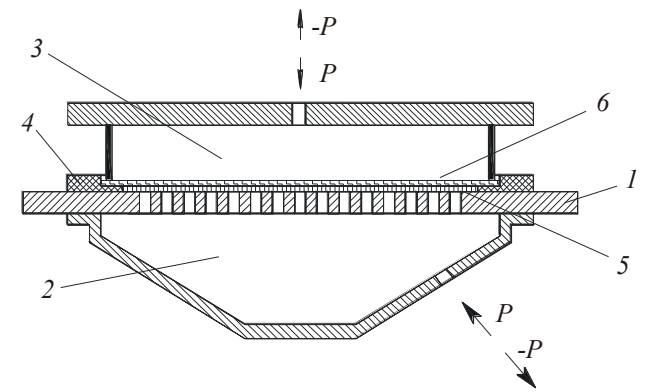

Figure 1 - Device for hide treatment: 1 - supporting slab; 2 - lower chamber; 3 - upper chamber; 4 - fixing frame; 5 - net; 6 - sample of hide

Operating principle: a sample 6 of the hide to be treated is placed on the net 5, which itself is on supporting slab 1 and the unhairing solution is poured on it. The sample is pressed from above by the fixing frame 4 and the upper chamber 3 . Air is continuously sucked from the lower chamber 2 . Due to the pressure difference, the unhairing solution is sorbed more intensively into the sample.

Method II: $10 \mathrm{ml}$ of the solution is poured on the subcutaneous side of sample (control) and distributed it over the whole area. The samples are placed in a polyethylene bag and kept for 0 to 12 hours. 
At regular intervals, the depth of absorption of sodium hydroxide and sodium sulphide into the derma, the strength of the bond between hair and dermis. The depth of absorption of the $\mathrm{NaOH}+\mathrm{Na}_{2} \mathrm{~S}$ solution into the hide is determined by staining the treated skin with phenolphthalein or nitroprusside indicator. The thickness of the section of hide stained with the indicator is then measured with a biological microscope with a micrometre scale.

The strength of the hair's bond to the derma is determined by a dynamometer with a special holder. Firstly, a $25 \times 50 \mathrm{~mm}$ specimen is cut from treated hide sample. In the middle of the specimen, a $3 \mathrm{~mm}$ wide section of hair is separated with a special needle. The remaining hair is cut off. The sample is then cut into five $10 \mathrm{~mm}$ wide strips. The strip is attached to the dynamometer as follows: the hair is carefully compressed into the upper dynamometer holder and the two cut ends of the strip into the lower one, which is $5 \mathrm{~mm}$ from the upper holder.

\section{Results and discussion}

It is known that due to the properties of the supermolecular structure of the hide, individual layers of the hide are not equally permeable to solutions - the most difficult solutions are to diffuse through the grain and flesh layers.

Therefore, we first investigated the effect of sample thickness on the depth of penetration of unhairing solution into the derma. Soaked hide samples of various thicknesses were treated with unhairing solutions in two ways: without (control samples) and with (test samples) quickening of the absorption of the solutions into the derma acting by vacuum. The specimens were placed subcutaneously in the device (Fig. 1) and the unhairing solution was poured on them. All samples were left to lie down after treatment with unhairing solution. The depth of penetration of the unhairing solution into the hide derma was determined after 0,$5 ; 3 ; 9$ and 12 hours of storage (Fig. 2).

The presented data show that the penetration of the unhairing solution after 0,5 hours is 2-3 times higher (Fig. 2, b) compared to the control samples (Fig. 2, a). During 12 hours, the unhairing solution penetrated completely through derma of samples (excepting 8,3 $\mathrm{mm}$ and thicker), for which the penetration of the solution was acted by vacuum. It is evident that the penetration of the unhairing solution through the dermis is accelerated by exposure to vacuum. 

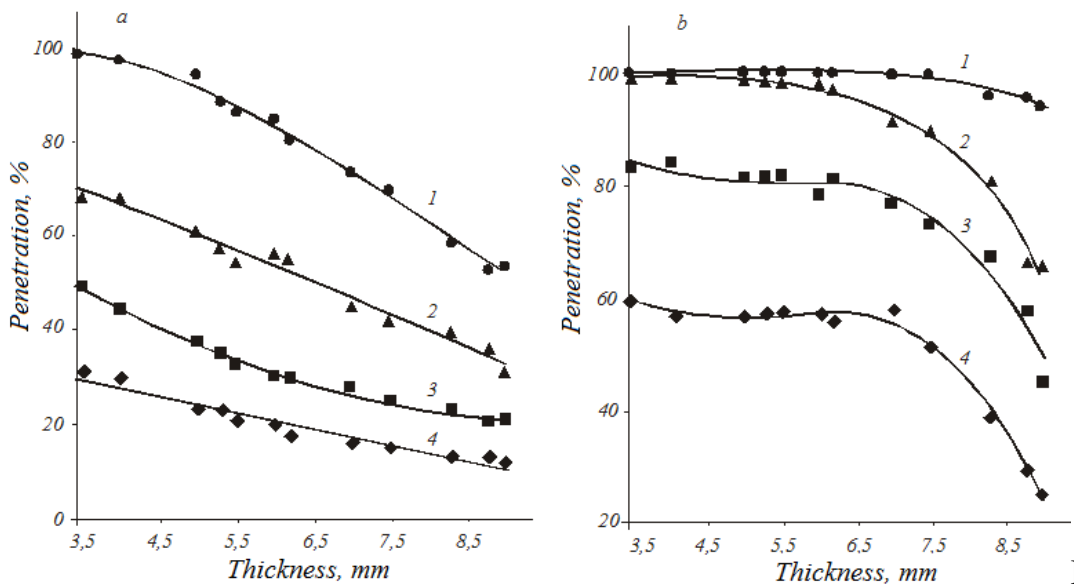

ure 2 - Dependence of $\mathrm{NaOH}+\mathrm{Na}_{2} \mathrm{~S}$ solution penetration on the thickness of samples, when storage time, hours: $1-12 ; 2-6 ; 3-3 ; 4-0.5$; $\mathrm{a}$ - control samples; $\mathrm{b}$ - test samples

The strength of the hair's bond with the derma determines the possibility of high-quality removal of hair from the hide by mechanical means. The force required to pull the hair out of the hide dermis is an indicator of the strength of the hair's bond to the derma. The samples (thickness 3,5; 5; 7 and $9 \mathrm{~mm}$ ) used to test the penetration of the unhairing solution were also examined to determine the strength of the bond between the hair and the dermis (Fig. 3).

The data on the change in the strength of the bond between hair and derma correlate with the data on the penetration depth of the unhairing solution (Fig. 3). Also, the mechanical hair removal from the hide can be performed qualitatively when a force of 0,98 to $1 \mathrm{~N}$ is sufficient to remove the $3 \mathrm{~mm}$ strip of hair.

Hence, after accelerating the penetration of the unhairing solution by vacuum, after 12 hours of treated sample storage the strength of the hair bond with the dermis decreases to $0,98 \mathrm{~N}$ in all cases, which means that the hair can be removed by dedicated mechanical means. 

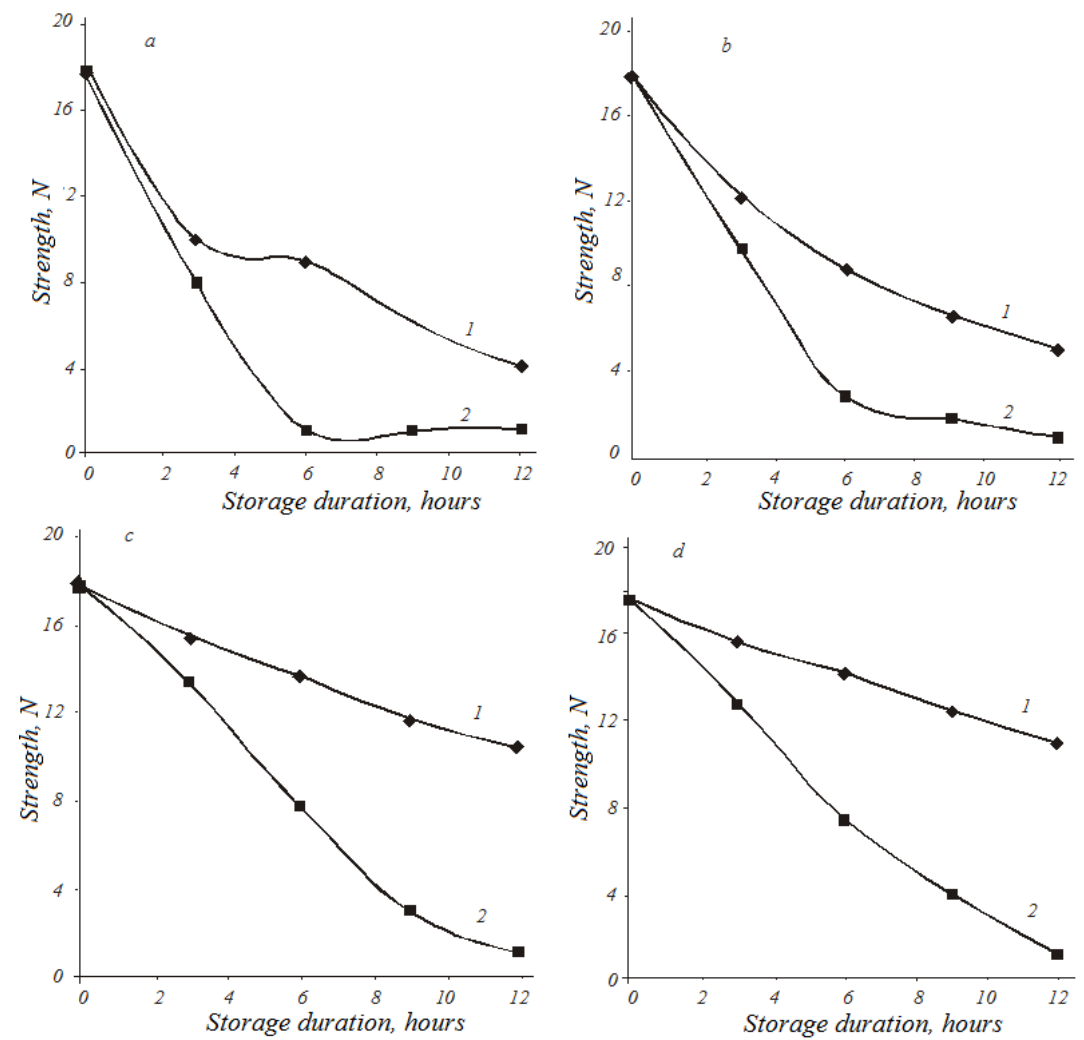

Figure 3 - Dependence of strength of hair's bond with derma on storage time at sample thickness, mm: $\mathrm{a}-3,5 ; \mathrm{b}-5,0 ; \mathrm{c}-7,0 ; \mathrm{d}-9,0$;

1 - control samples; 2 - test samples

\section{Conclusions}

The penetration of the unhairing solution into the hide can be accelerated by vacuuming. During 12 hours, the unhairing solution penetrated completely through derma of 3,5-7,5 mm thickness hide samples when the process was accelerated by vacuum. Meanwhile, without the use of a vacuum and under the same storage conditions, the penetration of the unhairing solution was $94-98,6 \%$ even in the thinnest control samples. After accelerating the penetration of the unhairing solution by vacuum, the strength of the hair's bond to the derma after 12 hours of storage does not exceed 0,98 
$\mathrm{N}$ in all cases, which means that the hair can be removed by mechanical means.

\section{References}

1. Sivakumar V. Influence of Ultrasound on the Adsorption, Diffusion and Kinetics of Leather Dyeing Process: Mechanistic Insight // Journal of the American Leather Chemists Association, 2020, 115, 7, P. 239-247.

2. Combalia F., Morera J.M., Bartoli E. Study of several variables in the fixation stage of a vegetable tannage after penetration under ultrasound // Journal of the American Leather Chemists Association, 2018, 113, 5, P. 134-141.

3. Mengistie E., Smets I., Van Gerven T. Ultrasound assisted chrome tanning: Towards a clean leather production technology. Ultrasonics Sonochemistry, 2016, 32, P. 204-212.

4. Chursin V.I., Lychnikov D.S. Investigation of the influence of an ultrasonic field on the process of chrome tanning // Textile chemistry, 1998, 3, 15, P. 8-10.

5. Goncharova V.S., Shestakova I.S., Ptuzhenekov L.N. Hydroacoustic method of processing leather raw materials // Leather and footwear industry, 1979, 9, P. 31-33.

6. Tricys V., Beleska K., Valeika V. Stimulation of the chrome tannage process by vacuum // Journal of the Society of Leather Technologists and Chemists, 2003, 87, P. 78-81.

7. Prokein M., Renner M., Weidner E., Heinen T. Low-chromium- and low-sulphate emission leather tanning intensified by compressed carbon dioxide. Clean Technologies and Environmental Policy, 2017, 19, P. 2455-2465.

8. Shapkhaev E.G., Trunin O.Yu., Dumnov V.S. Intensification of the tanning process by directed mechanical action // Leather and shoe industry, 2000,1 , P. 39.

9. Beschleunigte Technik bei der Entwollung // Leder, 1997, 48, 9, P. 198201. 